\title{
Estudio de un dispositivo tecnológico para la prevención de accidentes automovilísticos causados por ganado
}

\section{Study of a technological device for the prevention of automobile accidents caused by cattle}

ZÚÑIGA-MAYORAL, Kevin Jasiel†*, HERNÁNDEZ-VALENZUELA, Juan Carlos, MEZAARELLANO, Antonio y MEZA-ROSAS, Iliana Janeth

Instituto Tecnológico Superior de Mulegé. Centro, Loma los Frailes S/N, Centro, 23920 Santa Rosalía, B.C.S.

ID $1^{\mathrm{er}}$ Autor: Kevin Jasiel, Zúñiga-Mayoral

ID $1^{\text {er }}$ Coautor: Juan Carlos, Hernández-Valenzuela / ORC ID: 0000-0003-3523-232, Researcher ID Thomson: P-43442018, CVU CONACYT ID: 278735.

ID $2^{\text {do }}$ Coautor: Antonio, Meza-Arellano / ORC ID: 0000-0002-6553-8392, Researcher ID Thomson: P-6194-2018, CVU CONACYT ID: 404918

ID $3^{\text {er }}$ Coautor: Iliana Janeth Meza Rosas / ORC ID: 0000 0002-4173-2422, Researcher ID Thomson: P-5910-2018, CVU CONACYT ID: 931015

DOI: $10.35429 /$ JCA.2020.13.4.8.16

Recibido Enero 20, 2020; Aceptado Marzo 30, 2020

\section{Resumen}

En el presente artículo se muestra el análisis de la información adquirida en la investigación "Estudio para conocer la utilidad de un dispositivo tecnológico para la prevención de accidentes automovilísticos provocados por el ganado bovino en Santa Rosalía Baja California Sur", donde se recabaron los datos aplicando encuestas por vía electrónica utilizando las Tecnologías de la información y comunicaciones (TIC), De igual manera, se investigó el total de personas que poseen teléfonos celulares y su conocimiento sobre aplicaciones móviles, considerando que la finalidad de la investigación es determinar la viabilidad del dispositivo GPS que funciona en conjunto con la tecnología implementada en un teléfono celular, por consiguiente, con esto se permite crear estrategias para un mejor desarrollo y el correcto lanzamiento de dicha tecnología, obteniendo como resultado un $90 \%$ de aceptación para la implementación de un sistema de geolocalización de semovientes como alternativa de seguridad al trasladarse por la carretera transpeninsular dentro del estado.

Dispositivos tecnológicos, Móviles, Bovinos, Accidentes automovilísticos

\begin{abstract}
This article shows the analysis of the information acquired in the research "Study to know the usefulness of a technological device for the prevention of car accidents caused by cattle in Santa Rosalía Baja California Sur", where the data was collected by applying electronic surveys using Information and Communications Technologies (ICT), similarly, the total number of people who own cell phones and their knowledge of mobile applications, considering that the purpose of the research is to determine the viability of the GPS device that works in conjunction with the technology implemented in a cell phone, thus allowing to create strategies for a better development and the correct launch of such technology, resulting in $90 \%$ acceptance for the implementation of a system of geolocation of semovients as a safety alternative when moving along the transpeninsular road inside state.
\end{abstract}

Technology devices, Mobile, Bovine, Car accidents

Citación: ZÚÑIGA-MAYORAL, Kevin Jasiel, HERNÁNDEZ-VALENZUELA, Juan Carlos, MEZA-ARELLANO, Antonio y MEZA-ROSAS, Iliana Janeth. Estudio de un dispositivo tecnológico para la prevención de accidentes automovilísticos causados por ganado. Revista de Cómputo Aplicado. 2020. 4-13: 8-16.

\footnotetext{
* Correspondencia al Autor (Correo Electrónico: kevin.zuniga@itesme.edu.mx)

$\dagger$ Investigador contribuyendo como primer autor.
} 


\section{Introducción}

El Estado de Baja California Sur cuenta con una superficie de 73,677 kilómetros cuadrados, con una longitud de 750 kilómetros y una altitud promedio de 100 kilómetros; en este, se recorre la carretera federal 1, también conocida como carretera transpeninsular, que inicia en Cabo San Lucas y continua 998 kilómetros hasta el norte del municipio de Mulegé. A este Estado lo atraviesa en su largo una serranía, en la cual están ubicadas centenas de rancherías, en las que se dedican al cuidado y comercio de ganado, además de los productos elaborados u obtenidos a través de este. Las rancherías tienen grandes extensiones de tierra, las cuales muchas colindan o comparten espacio geográfico con el área de la carretera federal, por lo que es común encontrarse con ganado bovino, equino y en veces hasta caprino.

Se conoce que en el Estado hay leyes federales que protegen al conductor en caso se sufrir un accidente a causa de ganado suelto y que imponen al propietario a cercar sus tierras que coinciden con la carretera, pero también hay derechos que permiten al ganadero trabajar sobre toda la extensión de sus tierras; por esto, se sigue viendo presencia de estos animales incluso sobre el asfalto del trayecto, es cuando resulta un peligro para las personas que se trasladan de un lugar a otro por esta vía.

Por medio de diferentes investigaciones donde se presentan estadísticas obtenidas con trabajos junto a la Policía Federal, se dio a conocer que, en el año 2012 en el estado de Baja California Sur, hubo un saldo de 546 accidentes automovilísticos, en los cuales resultaron 60 personas muertas y 409 lesionados. Entre las causas más destacadas están el conductor, el camino, agentes naturales y el vehículo. Las causas atribuibles al conductor fueron la velocidad inmoderada, invasión de carril, imprudencia, malas condiciones físico-mentales, no respeto señal o dispositivo de tránsito entre otros. Dentro de las causas atribuidas al camino principalmente se encuentra la irrupción de ganado con más de $50 \%$ como causa, el resto está entre el pavimento mojado y objetos en el camino.
De los 546 accidentes dentro del estado en el año 2012, 20 fueron choques con semovientes, ocasionando pérdidas totales; mientras que otro registro del año 2017 menciona que el total de accidentes automovilísticos registrados dentro del estado fue de 201 y el $2.50 \%$ fue a causa de ganado.

Por esto, se realizó una investigación para conocer la aprobación en la sociedad de una tecnología compuesta por un dispositivo geolocalizador que sería instalable en el ganado y una aplicación móvil que pueda alertar a los conductores o personas que se encuentren viajando por la carretera, por la presencia de semovientes que pongan en peligro tanto su vida como la del animal.

*Se entiende por geolocalización como la capacidad de tener la ubicación en tiempo real de un objeto, animal o cosa.

\section{Metodología}

Se hará uso de los métodos de investigación exploratoria e inductiva a través de encuestas aplicadas dentro de la localidad de Santa Rosalía, utilizando medios electrónicos como el programa de Microsoft Forms, el cual permitirá obtener información y resultados acerca de la utilidad de un dispositivo tecnológico para la prevención de accidentes automovilísticos provocados por el ganado bovino, estas se realizarán a través de plataformas como correo electrónico, WhatsApp y Facebook, donde es importante obtener el número de los habitantes, la cual es de 14,160, de acuerdo con datos del (Lucero, 2015), que para ello es importante obtener la muestra a través de la siguiente formula:

$n=\frac{\mathrm{N} \sigma 2 \mathrm{Z} 2}{(N-1) e 2+\sigma 2 Z 2}$

(Suarez, 2011)

Donde $\mathrm{N}$ es el tamaño de la población, " $\sigma$ " es la desviación estándar de la población que, generalmente cuando no se tiene su valor, suele utilizarse un valor constante de 0.5 . " $\mathrm{Z}$ " representa al valor obtenido mediante niveles de confianza. 
Es un valor constante que, si no se tiene su valor, se lo toma en relación con el $95 \%$ de confianza equivalente a 1.96(como más usual) o en relación con el $99 \%$ de confianza equivale 2.58 , valor que queda a criterio del encuestador. "e" es el limite aceptable de error muestral que, generalmente cuando no se tiene su valor, suele utilizarse un valor que varía entre el 1\% (0.01) y $9 \%$ (0.09), valor que también queda a criterio del encuestador.

$n=\frac{(14160)\left(0.5^{2}\right)\left(1.90^{2}\right)}{\left((14160-1)\left(0.10^{2}\right)\right)+\left(\left(0.5^{2}\right)\left(1.90^{2}\right)\right)}=\frac{12779.4}{142.4925}=90$

Como resultado se obtuvo que: $n=90$.

\section{Resultados}

\section{Conocimiento de accidentes en la carretera por bovinos}

Actualmente la posesión y el uso de dispositivos electrónicos como teléfonos celulares, tabletas, televisores inteligentes, es cada vez mayor en la sociedad, ya que hay gran variedad de precios y facilidades de adquisición, estos dispositivos están al alcance casi de cualquier persona, brindando la capacidad de enviar y recibir mensajes o llamadas, así como de navegar en internet y hacer uso de redes sociales, es decir, las noticias e información pueden viajar de un lugar a otro casi instantáneamente, ahora bien, el estado de Baja California Sur, es uno de los estados más grandes del país, sin embargo, el número de la población es muy baja en comparación a otros estados, enterarse y conocer casos de accidentes ocasionados por semovientes a través de notas periodísticas o a través de mensajes por personas alrededor del usuario no debería ser difícil o de posibilidad lejana, ya que es algo que debe estar en la actualidad al alcance de todos, tal como lo expresa (Martínez, 2014) comenta que hoy no solo se utiliza el teléfono como un dispositivo para hacer llamadas, sino que se puede consultar agendas, pronóstico, geolocalización y navegación por internet entre otras actividades.

Un estudio realizado en Santa Rosalía, demuestra que el $100 \%$ de los encuestados tiene conocimiento acerca de la ocurrencia de accidentes ocasionados por semovientes que se sitúan sobre la carretera dentro del estado, siendo el $60.44 \%$ del sexo femenino y $38.46 \%$ del sexo masculino.

\section{Conocimientos de accidentes por parte de los usuarios}

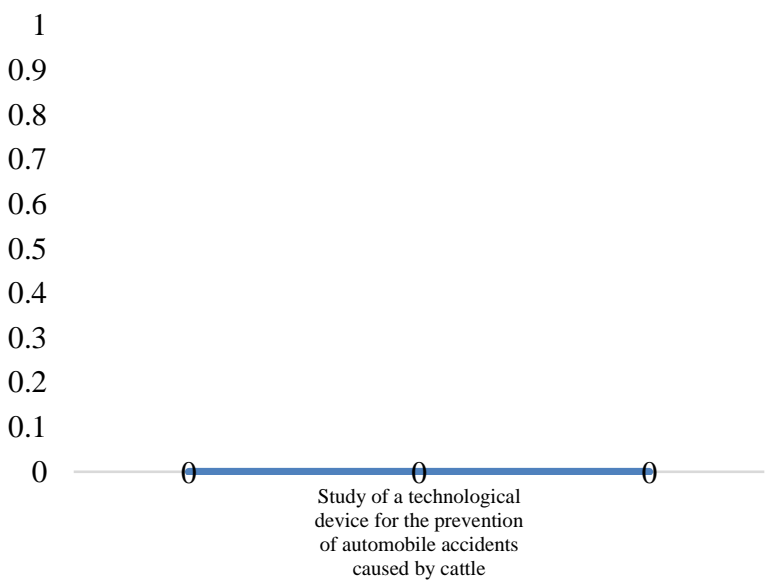

Gráfico 1 Conocimiento de accidentes por parte de los usuarios; fuente de elaboración propia; tomada del estudio: Utilidad de dispositivos tecnológicos para la prevención de accidentes automovilísticos en Santa Rosalía, Baja California Sur

Es de suma importancia conocer la existencia de ocurrencia de accidentes por causa de semovientes, ya que muchas veces se corre el riesgo de presentarse en una situación ocasionada por este motivo. Gracias a las tecnologías de la información y comunicaciones (TIC), es de mayor facilidad enterarse de noticias y alertas de presencia de ganado sobre la carretera en determinadas zonas o kilómetros entre lugares dentro del estado de Baja California Sur, lo cual, si existiera una tecnología capaz de mostrar una alerta cuando un semoviente, previamente registrado por el fabricante del dispositivo, estuviese cerca de la ubicación del vehículo, podría disminuir la probabilidad de tener un accidente.

\section{Víctimas de accidentes ocasionados por irrupción de ganado}

Es una realidad que, dentro del estado de Baja California Sur, ocurren accidentes automovilísticos con frecuencia, siendo el conductor el principal causante de estos, como también el peatón o pasajero, otros vehículos, el camino, por irrupción de ganado o agentes naturales. 
La mayoría de los accidentes ocurren dentro de las ciudades del estado, como La Paz o Cabo San Lucas, Cd. Insurgentes y Loreto, sin embargo, a lo largo de la carretera transpeninsular, hay tramos donde es común encontrarse con semovientes sobre la carretera o cerca a esta, ya que en las orillas del asfalto crece vegetación con la cual estos animales se alimentan y a pesar de la existencia de letreros y señalamientos, muchas veces el ganado bovino se establece en tramos que no están marcados de su posible presencia, resultando una sorpresa para el conductor que transita por la carretera. Ahora bien, a través del tiempo, ha habido personas que desafortunadamente han sufrido accidentes por esta causa, resultando en daños materiales ocasionados por el impacto con el animal, como lesiones en el cuerpo del o los pasajeros a bordo del vehículo o incluso la muerte por ambas partes, incluyendo al semoviente. Por otra parte, es común que otras personas se enteren de estos acontecimientos, ya sea por estar cerca del lugar de los hechos, ser parte del suceso o estar vinculados familiarmente o ser allegados de las víctimas, a través de noticias, reportes policiacos, llamadas o mensajes, notas en redes sociales, entre otros medios de comunicación. El sitio web (gob.mx, 2018), expone las causas de los accidentes automovilísticos, así como los lugares con mayor ocurrencia además del saldo de heridos y muertes.

De acuerdo con un estudio realizado en Santa Rosalía, de manera online, señala que el $24.44 \%$ de los encuestados ha sufrido un accidente, además, el $16.67 \%$ no conoce a ninguna persona que haya tenido un imprevisto por ganado, por otro lado, el $75.56 \%$ afirmó que no ha sido parte de un accidente por semovientes, sin embargo, el $83.33 \%$ de los encuestados dice que sí conocen a personas que han padecido un percance por irrupción de ganado.
Victimas de accidentes ocasionadas por irrupción de ganado

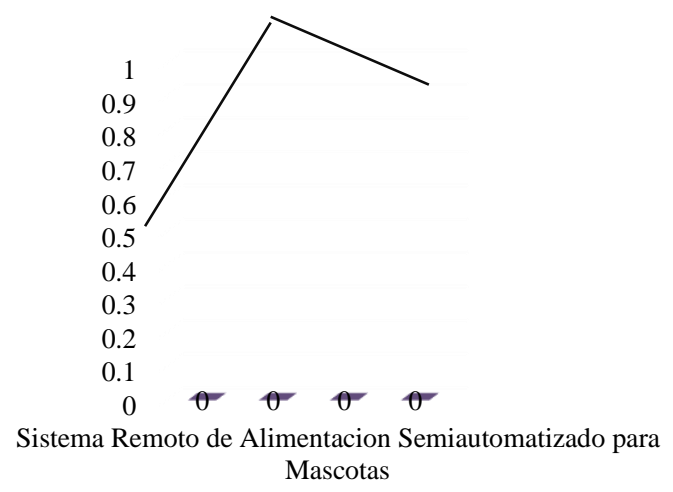

Gráfico 2 Víctimas de accidentes ocasionados por irrupción de ganado; fuente de elaboración propia; tomada del estudio: Utilidad de dispositivos tecnológicos para la prevención de accidentes automovilísticos en Santa Rosalía, Baja California Sur.

En definitiva, la ocurrencia de accidentes dentro del estado de Baja California Sur, es algo serio, la pérdida de vidas humanas y de los animales involucrados es lamentable, por eso es importante estar enterados de las zonas con presencia de ganado, además de cumplir con las normas de seguridad al conducir, se debe prestar atención a los señalamientos, hay tramos donde no hay letreros, sin embargo, no se debe descargar el hecho de poder encontrar sin previo aviso, alguna irrupción por ganado, el cual pueda ocasionar una tragedia. Con la implementación de una tecnología capaz de señalar donde hay semovientes cercanos o que estén sobre la carretera, se pudiera disminuir la posibilidad de tener un accidente.

\section{Necesidad de implementar una medida de seguridad}

Los ciudadanos del estado de Baja California Sur están enterados de la ocurrencia de accidentes provocados por la irrupción de ganado sobre la carretera. La búsqueda de alternativas de seguridad para saber la ubicación de estos animales sobre la vía transpeninsular no ha sido muy atendida. Es cierto que se han establecido propuestas realizadas por diferentes diputados dentro del estado, para agravar sanciones a los ganaderos que tengan animales en la carretera, con multas por miles de pesos, no obstante, en Baja California Sur, el libre pastoreo es un acto respaldado por la ley estatal, por esto el método de cercar las orillas de las carreteras entra en conflicto con los derechos de los ganaderos resultando en un difícil debate. 
Se reconoce que para lograr esto, ambos lados tendrían que ceder, una realidad es que el ganadero aplica una fuerte inversión para el cuidado de sus animales, aplicar sanciones de alta cantidad monetaria podría resultar no conveniente, ya que muchas veces el propietario del semoviente no tiene recursos económicos para cubrir una multa por este motivo. Por otro lado, también se ha buscado señalar al ganado con cintas luminosas para poder ver al animal contra luz durante la noche, a pesar de esto, el método no ha funcionado de manera exitosa, ya que el animal puede encontrarse en una posición no favorable para la reflexión de la luz, como también el desprendimiento de la cinta. Es por esto por lo que, las personas expresan la necesidad de aplicar un método que les brinde seguridad a la hora de trasladarse por carretera. (Martínez, 2014) comenta que hoy en día la comunicación a través del celular ha dejado de ser única función, ahora se pueden realizar diversas actividades con el dispositivo, como la geolocalización.

Con respecto a un estudio realizado en Santa Rosalía, se obtuvo que, primeramente, el $100 \%$ saben que en el estado hay accidentes ocasionados por semovientes, a su vez, el $97.78 \%$ de los encuestados cree que se necesita implementar un método que brinde seguridad al trasladarse sobre la carretera y tan solo el $2.22 \%$ mencionó que no es relevante implementar un método de seguridad.

\section{Necesidad de implementar una medida de seguridad}

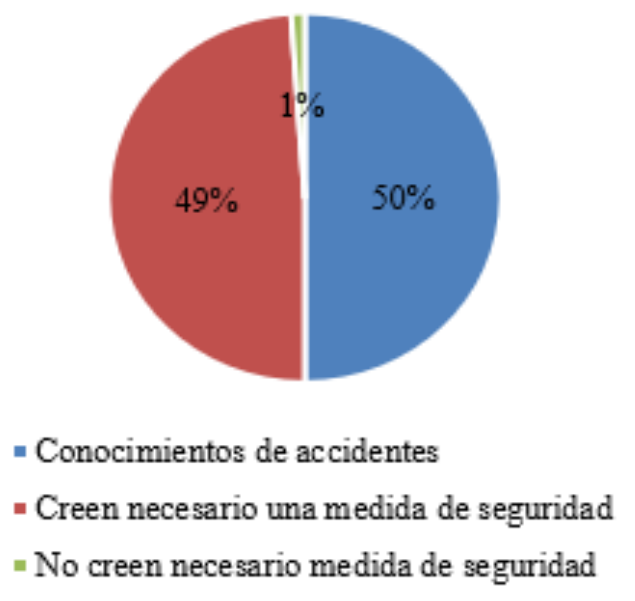

Gráfico 3 Necesidad de implementar una medida de seguridad; fuente de elaboración propia; tomada del estudio: Utilidad de dispositivos tecnológicos para la prevención de accidentes automovilísticos en Santa Rosalía, Baja California Sur
Los ciudadanos están conscientes de que en la carretera puedes sufrir algún imprevisto y de estos, pueden ser ocasionados por una irrupción de ganado, a pesar de que no todos los encuestados han pasado por una situación de accidente, creen que es importante aplicar un método que brinde seguridad al trasladarse por los tramos de carretera dentro del estado de Baja California Sur. Si hubiese una tecnología capaz de alertar al viajero cuando hay una vaca cerca, es muy probable que las personas deseen obtenerla y utilizarla, ya que han afirmado creer necesario una medida de seguridad.

\section{Uso del teléfono celular}

Algunas décadas atrás, poseer un teléfono celular era un lujo que no cualquier persona podía tener, era solo para los que realmente lo necesitaban y, además, podían comprar. Hoy en día hablar de teléfonos móviles quizás ya no es novedoso, esto es porque la mayoría de las personas en nuestra sociedad alrededor del mundo conoce sobre la existencia, el uso y las herramientas digitales que nos ofrecen, Podría decirse que estos aparatos electrónicos ya son parte de la vida cotidiana, pues en ellos se puede encontrar un medio de comunicación, a través de llamadas de voz o video, mensajes de audio y texto, entretenimiento con miles de aplicaciones y acceso a internet, la función de tomar fotografías y grabar videos permitiendo compartirlos en redes sociales, entre otras tantas posibilidades. El número de personas que tienen un celular se estima en 4,770,000,000, comparándolo a las 7,400,000,000 personas que viven en el mundo, casi un $64 \%$ de la población en el mundo tiene un teléfono celular, además se sabe que cerca del $83 \%$ del acceso a internet se hace desde teléfonos móviles en el mundo. De acuerdo con una encuesta realizada por (INEGI, 2017), 81 millones de personas en México tienen acceso a un teléfono celular y de estos, el 74.8 por ciento adquirieron un teléfono inteligente $\mathrm{o}$ smartphone, lo que indica que al menos tres de cada cuatro usuarios cuentan con un dispositivo de este tipo. Con respecto a un estudio realizado en Santa Rosalía, de manera online, se analizó que durante los últimos 3 años del presente 2018, el aumento de la posesión y uso del celular fue de un $2.22 \%$ en la población, siendo un $96.67 \%$ 3 años atrás y actualmente un $98.89 \%$ las personas que tienen un teléfono móvil. Tan solo el $1.11 \%$ del total, no tiene actualmente este dispositivo. 
Uso del telefono celular

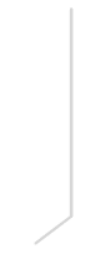

0

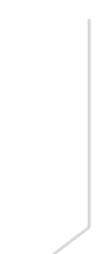

0.5

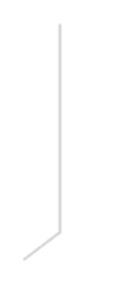

1

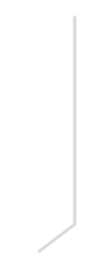

1.5

Gráfico 4 Uso del teléfono celular; fuente de elaboración propia; tomada del estudio: Utilidad de dispositivos tecnológicos para la prevención de accidentes automovilísticos en Santa Rosalía, Baja California Sur

El aumento en la posesión y uso del teléfono celular de las personas encuestadas es de al menos un $2.22 \%$ en los últimos 3 años, siendo evidente la casi total posesión del dispositivo móvil por todos los usuarios, con esto se puede concluir que sería factible el implementar una tecnología utilizando este medio.

\section{Conocimiento acerca de aplicación móvil}

Se conoce que la mayoría de las personas alrededor del mundo cuentan con un teléfono celular, utilizándolo como herramienta diaria para realizar diferentes actividades, como comunicarse o realizar compras en línea. Para que un teléfono celular pueda ser manipulado por un usuario, este necesita tener instalado un sistema operativo, el cual es un conjunto de órdenes y programas que controlan los procesos básicos del celular, es lo que nos permite ver el contenido en el móvil, y sobre este, se instalan las aplicaciones o "apps" que los usuarios utilizan, como Facebook, Whatsapp, Instagram, entre miles más. Sin embargo, no todas las personas que tienen un teléfono móvil conocen el termino o el concepto de aplicación, lo que resulta de mucha importancia al hablar de una tecnología de rastreo, la cual está compuesta por una app. (Navarro, 2014) comenta que una aplicación (también llamada app) es simplemente un programa informático creado para llevar a cabo o facilitar una tarea en un dispositivo informático.

Con respecto a un estudio realizado en la localidad, de manera online se obtuvo que un $98.89 \%$ de los encuestados, actualmente cuenta con un teléfono celular, por otro lado, el $20.00 \%$ mencionó que no conoce el termino de aplicación móvil.

\section{Conocimiento acerca de la aplicación} movil

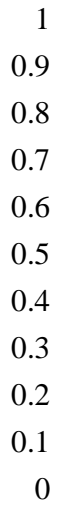

Gráfico 5 Conocimiento acerca de aplicación móvil; fuente de elaboración propia; tomada del estudio: Utilidad de dispositivos tecnológicos para la prevención de accidentes automovilísticos en Santa Rosalía, Baja California Sur.

Actualmente la mayoría de las personas tienen un teléfono celular, sin embargo, no todos conocen el termino de aplicación móvil o aplicación en el celular. A pesar de utilizar dichas aplicaciones, estos desconocen al menos su concepto, como tal donde es importante recalcar que cuando al usuario le explicas que es, te lo entiende una mejor manera y se familiariza con dicha herramienta.

\section{Utilizar una aplicación de geolocalización}

Con los avances de la ciencia y la tecnología, se han desarrollado una infinidad de dispositivos electrónicos que le ayudan al ser humano a mejorar su calidad de vida, un ejemplo son los sistemas de GPS, los rastreadores para localizar objetos, personas y cualquier cosa al que se le pueda instalar un pequeño aparato el cual revelaría su posición al usuario. Hay muchos usos que se le puede dar a esta tecnología, como entretenimiento, exploraciones, investigaciones, para uso industrial entre otras. Hay dos tipos de rastreo satelital, el pasivo y el activo, el pasivo trabaja fuera de línea, por lo regular recibe y muestra la información en el mismo aparato, los activos son los que trabajan en línea, estos pueden recibir y enviar a un receptor los datos que recaba, por ejemplo, en un celular a través de una aplicación. La empresa (Max4, 2009) menciona que el Rastreo Satelital es un servicio que permite localizar vehículos, personas u objetos en cualquier parte del mundo por medio de triangulación de señales emitidas por 27 satélites geoestacionarios alrededor del planeta. 
Con respecto a un estudio realizado en Santa Rosalía, de manera online se recabó que el $61.11 \%$ de las personas encuestadas, sí utilizarían una aplicación de rastreo, el $23.33 \%$ señaló que no la utilizaría mientras que el $15.56 \%$ tal vez lo haría.

\section{Utilizar una aplicación de geolocalización}

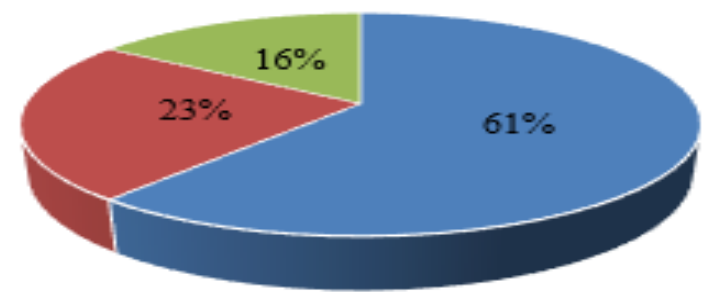

- Utiliza una aplicación movil que le mostrala la ubicación de un animal bovino

- No utilizaria la aplicación

- Tal vez Utilizaría la aplicación

Gráfico 6 Utilizar una aplicación de geolocalización; fuente de elaboración propia; tomada del estudio: Utilidad de dispositivos tecnológicos para la prevención de accidentes automovilísticos en Santa Rosalía, Baja California Sur

A pesar de que una proporción elevada de los encuestados mencione que no utilizaría una aplicación como esta, la mayoría de ellos, y sumando a los que probablemente lo harían, da como resultado un numero positivo para una posible implementación de la mencionada tecnología.

\section{Precios dispuestos a pagar}

Hoy en día, las compras en línea son cada vez más frecuentes, ya que es un método que está al alcance de cualquier persona y su proceso es muy fácil de realizar. En el mercado on-line se puede encontrar una infinidad de productos y servicios que se pueden adquirir por medio de un pago, ya sea por transferencia bancaria o a través de una tarjeta de crédito o débito. En el mundo de la telefonía móvil, existen 2 grandes tiendas de aplicaciones para el celular que son ofrecidas por Apple y Android, cada una para sus determinados sistemas operativos; dentro de las tiendas, las aplicaciones se dividen en diferentes categorías y precios, hay gratuitas y de pago.
Dependiendo la utilidad, funcionalidad, complejidad y novedoso de la aplicación, será el costo que tenga establecido, regularmente las aplicaciones tienen una versión gratuita y otra con costo. (Mencía, 2017) menciona que una opción es establecer nuestro precio de venta en función de los precios de las otras apps de nuestro sector. De esta manera, una vez analizados los precios, podemos subir el precio un poco para denotar calidad.

Ahora bien, con respecto a un estudio realizado en Santa Rosalía, de manera online, se demostró que el $100 \%$ de los encuestados está dispuesto a pagar por una aplicación de rastreo de bovinos sobre la carretera, siendo el $68.00 \%$ los que están dispuestos a pagar entre $\$ 50$ y $\$ 100$ pesos, el $22 \%$ entre $\$ 100$ y $\$ 200$ pesos, mientras que el $5.33 \%$ pagaría entre $\$ 200$ y $\$ 500$, el $4.00 \%$ entre $\$ 500$ y $\$ 1000$.

\section{Precio dispuestos a pagar}

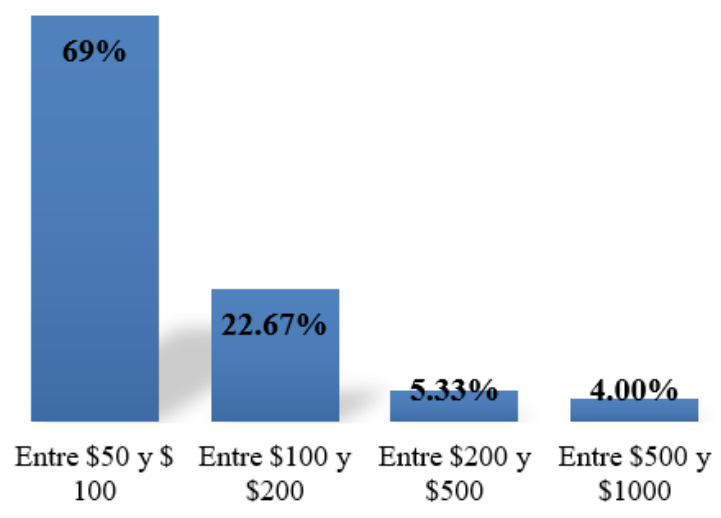

Gráfico 7 Precios dispuestos a pagar; fuente de elaboración propia; tomada del estudio: Utilidad de dispositivos tecnológicos para la prevención de accidentes automovilísticos en Santa Rosalía, Baja California Sur

Al desarrollar una aplicación móvil, se toma en cuenta el cliente, modelo de negocio, utilidad, promoción y el marketing. No hay una formula exacta para calcular el precio que se le asigna a una aplicación móvil, sin embargo, se toman en cuenta la mano de obra, la tecnología que se utiliza, el tiempo y la complejidad del desarrollo, entre otras cosas. El cliente final es un elemento importante para establecer el precio, usando un precio variable del cual se puede percibir la aceptación y la demanda que generará, es cuando entonces, se puede lograr un costo final. 


\section{Tecnología como seguridad al conducir por carretera}

Es común que, a lo largo de la carretera transpeninsular dentro del estado de Baja California Sur, se pueden encontrar semovientes situados sobre el camino a distancias del carril significativamente peligrosas. A pesar de la existencia de señalamientos, con advertencia de ser zonas con cruce de ganado, hay casos donde se han sufrido accidentes por diferentes factores, como pueden ser climatológicos, con neblinas, lluvia, etc., que dificulten la visión sobre la carretera. Hay zonas donde no hay señalamientos de advertencia de cruce de ganado a pesar de la presencia de estos. En la actualidad la tecnología ha avanzado a pasos agigantados, no obstante, para este tema y en la región del municipio de Mulegé, la tecnología está muy limitada. Por otro lado, hay vehículos de modelos recientes que cuentan con dispositivos para captar obstáculos en el camino, además de sistemas de aceleración controlada para reducir la velocidad y evitar tragedias. En el sitio web Motor16, (Sánchez, 2015) menciona que la automotriz "Ford", tiene en desarrollo un sistema que es capaz de localizar de forma simultánea hasta ocho personas y animales de cierto tamaño, como perros, en un radio de hasta 120 metros. Con respecto a un estudio realizado en Santa Rosalía, de manera online, se logró identificar a la porción de los encuestados que creen que la tecnología propuesta es útil hoy en día, siendo la mayor cantidad con el $95.56 \%$, mientras que solo un $4.44 \%$ piensa que esta tecnología no sería útil actualmente.

\section{Tecnológia como seguridad al conducir por carretera}

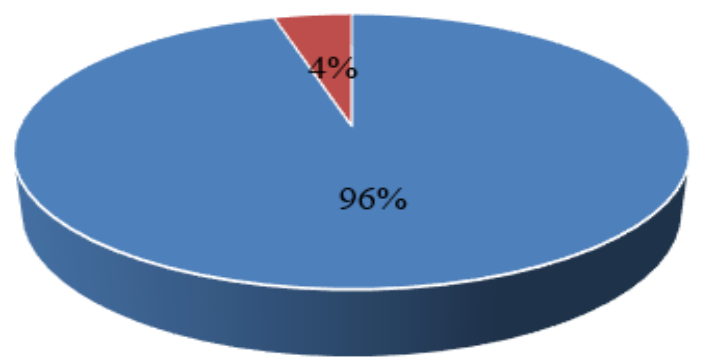

- Creen que la tecnológia de rastreo es útil hoy en dia

- No creen que la tecnologia sea útil hoy en dia

Gráfico 8 Tecnología como seguridad al conducir por carretera; fuente de elaboración propia; tomada del estudio: Utilidad de dispositivos tecnológicos para la prevención de accidentes automovilísticos en Santa Rosalía, Baja California Sur
Teniendo en cuenta la existencia de irrupción por ganado sobre la carretera federal en el estado, efectuar un método que apoye a disminuir el peligro al transitar por esta vía puede decirse que no solo se trata de un lujo, ya que se trata de la vida misma de las personas y del animal del que se posibilita el riesgo de perder.

\section{Propuesta}

Llevar a cabo la implementación del dispositivo geolocalizador y la aplicación móvil, para ofrecer un servicio que funcione como alternativa de seguridad a la hora de transitar o viajar por la carretera transpeninsular del Estado de Baja California Sur, para evitar o disminuir la probabilidad de ocurrencia de accidentes por irrupción de ganado. Dando al usuario la posibilidad de adquirir una aplicación que pueda instalar en su teléfono celular, desde la tienda online de aplicación móviles que le ofrece su versión de dispositivo, siendo posible obtenerla con un bajo costo que esté al alcance de cualquier usuario interesado.

\section{Recomendaciones}

- Desarrollar y ejecutar el lanzamiento del dispositivo de rastreo, utilizando las mejores herramientas tecnológicas para alcanzar el propósito establecido que es el prevenir accidentes automovilísticos.

- Desarrollar y lanzar al mercado digital la aplicación móvil disponible primeramente para usuarios de telefonía Android.

- Ofrecer dos versiones de la aplicación, una gratuita con función limitada y otra con costo que incluye funcionalidades de interés $\mathrm{y}$ apoyo al usuario.

- Definir el costo de la aplicación tomando en cuenta la elección de las personas encuestas $\mathrm{y}$ las estrategias de marketing actuales.

- Dar publicidad a esta tecnología para que esté al alcance de todas las personas, entre más grande sea la red del sistema de geolocalización, más seguridad se podrá brindar a los usuarios. 
- Estar profesionalmente comprometidos con los usuarios y consumidores de esta tecnología, en proteger la información o datos que se recaben por el sistema de rastreo para brindar seguridad y confianza.

\section{Conclusiones}

La sociedad al presente está informada y consciente de la ocurrencia de accidentes a lo largo del Estado de Baja California Sur sobre la vía de comunicación asfáltica, ocasionada por la irrupción de ganado y porcentualmente, una gran parte de ésta viaja de manera poco o muy frecuente a otros lugares dentro del estado por medio de vehículos terrestres, lo que los pone en riesgo de tener un percance de este tipo en su trayecto. Si bien, la mayoría de las personas son propietarios de un teléfono celular, no todos están enterados del concepto de "aplicación móvil" a pesar de que estas se utilizan diariamente para hacer funcionar su dispositivo. Sin embargo, para la sociedad, el celular es una herramienta muy importante hoy en día porque logra la comunicación inalámbrica a distancia y el poder tener conocimiento de lo que pasa alrededor del mundo. Una función que también incluyen los celulares es utilizar su sistema de posicionamiento global (GPS), el cual, al trabajar en conjunto con otros módulos digitales y herramientas tecnológicas, puede convertirse en una herramienta muy útil, así surgió la idea de implementar la tecnología que fue propuesta como alternativa para la seguridad de las personas que viajen sobre la carretera, siendo una idea aceptada con disposición de ser adquirida y hasta pagada por los usuarios.

\section{Referencias}

Navarro, R. L. (2014). Desarrollo de aplicaciones móviles. Perú.

Gob.mx. (2017). Estadística de Accidentes de Tránsito. Recuperado el 22 de noviembre de 2018, http://www.sct.gob.mx/carreteras/direcciongeneral-de-servicios-tecnicos/estadistica-deaccidentes-de-transito/
Lucero, H.L. (2015). Secretaria de Salud de Baja California Sur, Instituto de Servicio de Salud. Dirección y planeación de desarrollo, subdirección de planeación y estadística. Recuperado el 22 de noviembre de 2018, de http://www.saludbcs.gob.mx/estadistica/Tarjeta s\%20Estadisticas\%20Ejecutivas\%202015.pdf

Martínez, R. M. (2014). Formación del profesorado en la sociedad digital. Madrid: Universidad Nacional de Educación a Distancia. INEGI. (2017). Encuesta Nacional sobre Disponibilidad y Uso de Tecnologías de la Información en los Hogares 2017. Recuperado el 22 de noviembre de 2018, de http://www.beta.inegi.org.mx/proyectos/enchog ares/regulares/dutih/2017/

Max4. (2009). Max4 Technologies. Recuperado el 22 de noviembre de 2018, de http://www.max4systems.com/rastreosatelital.html

Mencía, J. M. (13 de octubre de 2017). Con tu negocio. ¿Qué precio pongo a mi aplicación móvil? Recuperado el 22 de noviembre de 2018, de

https://www.contunegocio.es/tecnologia/queprecio-pongo-a-mi-aplicacion-movil/

Sánchez, J. M. (22 de Julio de 2015). Motor16. Ford, desarrollando la iluminación del futuro. Recuperado el 22 de noviembre de 2018, de https://www.motor16.com/tecnologia/forddesarrollando-la-iluminacion-del-futuro/

Suarez, M. (2011). Estadística Básica. Obtenido de Interpretando la Estadística Básica: http://www.mat.uda.cl/hsalinas/cursos/2011/ey p2/Clase1.pd 